\title{
Centros de Integración Juvenil 1969-2019: 50 años, historia, retos y perspectivas
}

\author{
Carmen Fernández Cáceres \\ Directora General. Centros de Integración Juvenil, A.C.
}

Hace 50 años, el consumo de drogas legales e ilegales no presentaba las características, dimensiones y problemáticas asociadas con la violencia y criminalidad que hoy tenemos.

El consumo de sustancias se manifestaba fundamentalmente en segmentos poblacionales focalizados, identificados, y no tenía grandes repercusiones sociales y sanitarias de riesgo público.

La realidad desbordó y tomó por sorpresa a los especialistas de la salud, a la sociedad y al gobierno.

Asimismo, el acto del consumidor socialmente se consideraba más cercano a la comisión de un delito, a un "vicio", a la expresión de una "fractura moral", que a un comportamiento relacionado con la dinámica del complejo social y de la salud mental.

En un contexto general y como aproximación para el análisis, el grupo poblacional de riesgo en los años 60 y 70 del siglo pasado, en Norteamérica, Sudamérica, Europa y México, eran los jóvenes de los sectores urbanos, tanto populares como de los estratos medios. Esta tendencia se ha mantenido durante 50 años.

La emergencia de la coyuntura de ese entonces requirió de la participación social y política de su componente más dinámico y formado: los jóvenes de las ciudades.

El control de este grupo social siempre estuvo fuera del alcance total de los gobiernos occidentales. La movilización del segmento intelectual joven de la sociedad urbana expresó fuertemente su descontento contra el estado de cosas.

"Prohibido prohibir, seamos sensatos, pidamos y construyamos lo imposible".

Postulados todos ellos, aparecidos en las paredes de las principales ciudades del mundo, que fijaban una postura contestataria ante la autoridad gubernamental. La embriaguez de los jóvenes de saberse y auto descubrirse como fuerza política, social y cultural de cambio real.

En este marco global de revuelta, rebelión e inclusive revolución en Occidente, nace en México el Centro de Trabajo Juvenil, posteriormente Centros de Integración Juvenil, como un espacio de atención terapéutica y de rehabilitación de jóvenes consumidores de drogas.

Esta institución fue diseñada como punto de encuentro de jóvenes, donde se compartían experiencias de vida y habilidades técnicas, manuales e intelectuales.

En este espacio de trabajo se desarrollaban actividades artesanales, artísticas, culturales, educativas y recreativas. Bajo el modelo de abordaje de ese entonces, se planteaba que, a través de estas actividades, se reforzaban los factores protectores articulados a la prevención, rehabilitación y reinserción

Después del 68, en México se vivía un ambiente de desconfianza generalizada y una efervescencia social sin parangón en la historia contemporánea del país. En ese contexto, Centros de Integración Juvenil fue aceptado por los jóvenes que utilizaron nuestros servicios.

El origen fundacional de la institución y su propuesta inclusiva de trabajo ganaron la confianza de nuestra población objetivo: los jóvenes mexicanos.

Durante 50 años de trabajo ininterrumpido en la atención integral del consumo de drogas, ClJ ha consolidado estrategias de intervención flexibles que se han adaptado a la dinámica del complejo social y en éste, a las múltiples facetas que las juventudes mexicanas han presentado a lo largo de todos estos años.

Promovemos la salud física y mental, teniendo como continente, las posibilidades, problemas y oportunida-

\footnotetext{
Autor de correspondencia:

Mtra. Carmen Fernández Cáceres. Centros de Integración Juvenil, A.C. Av. San Jerónimo núm. 372, Col. Jardines del Pedregal, C.P. 01900, Ciudad de México, México. Teléfono: (0155) 528695 97. Correo electrónico: cfernandez@cij.gob.mx doi: 10.28931/riiad.2019.1.01
} 
des que dan las dinámicas familiares y sociales de los municipios, estados y regiones del país donde tenemos presencia.

Diseñamos e instrumentamos programas y acciones dirigidos a niños, niñas, jóvenes, padres de familia, maestros, mujeres, representantes institucionales, líderes de opinión, legisladores, obreros, campesinos, migrantes y grupos vulnerables, entre otros, a fin de ampliar nuestros impactos y extender la red de atención de adicciones existente en México.

A lo largo de todo este tiempo, hemos colaborado con todas las administraciones federales, estatales y locales, bajo la premisa de trabajar coordinadamente en favor de la salud de los mexicanos.

Hoy, en 120 puntos estratégicos del país, desarrollamos programas preventivos, de tratamiento y rehabilitación de las adicciones, así como proyectos de investigación y acciones formativas y de capacitación de recursos profesionales en materia de atención integral del consumo de drogas, en cualquiera de los escenarios de su compleja expresión.

En tal sentido, procuramos leer y entender la realidad y dinámica de las adicciones, según las manifestaciones particulares de su expresión, para así estar en condiciones objetivas de intervenir en ellas con oportunidad y eficacia.

Generamos, así, información científica con el fin de dar sustento a nuestros programas; en años recientes, hemos hecho un esfuerzo para contribuir a la difusión del conocimiento en la materia, por medio de la publicación de esta Revista Internacional de Investigación en Adicciones.

A lo largo de los años, la labor de investigación de ClJ ha estado vinculada y enfocada en apoyar, con sustento científico, la toma de decisiones relativas al diseño, planeación y ajuste de los programas sustantivos de prevención y tratamiento del uso de drogas.

En nuestro primer decenio, colaboramos con diversas instituciones para comenzar una etapa de exploración y reconocimiento del problema del uso de drogas y las adicciones, con énfasis en la investigación clínica y epidemiológica. En el segundo, formalizamos campos de estudio y recursos metodológicos; adoptamos, además, un enfoque basado en la medicina social y la salud pública, lo cual logró sistematizar los estudios epidemiológicos y proyectos con un enfoque de "investigación-acción".

Durante la tercera década de existencia institucional, desarrollamos múltiples estudios de corte empírico-deductivo y dimos un fuerte impulso a la investigación psicosocial, en particular mediante el desarrollo de modelos de riesgo. En los 10 años siguientes, ampliamos la cobertura del programa de investigación, a través de la diversificación de temas, poblaciones de estudio y perspectivas teóricas.

En los últimos años, hemos delineado una serie de temas prioritarios de estudio: migración, sexualidad, género, violencia y exclusión, comorbilidad y patología dual y nuevas sustancias de abuso. Propugnamos, además, por la aplicación de diseños de investigación que garanticen mayor validez y, con ello, aporten información más sólida para el desarrollo de programas y alternativas de atención. Hemos impulsado, además, la colaboración con institutos nacionales de salud e instituciones de educación superior.

De manera similar, nuestro trabajo se ha visto fortalecido, en virtud de las alianzas estratégicas que hemos convenido con instituciones gubernamentales, organizaciones civiles y empresas privadas, cuyas actividades son afines y complementarias a nuestra misión institucional.

Asimismo, procuramos la participación y el apoyo de la población, a fin de que los programas y proyectos que implementamos cuenten con el soporte de la comunidad.

En esta línea, nuestras unidades operativas son parte de la cotidianidad de cada colonia y ciudad donde nos encontramos. Cada unidad fomenta el establecimiento de redes interinstitucionales e intersectoriales de atención, de tal suerte que las acciones se entretejen para evitar la atomización de esfuerzos y el desperdicio de recursos.

México vive hoy tiempos muy complejos y de gran riesgo para nuestro futuro como nación. El consumo de sustancias durante los últimos 10 años ha crecido de manera importante. La violencia asociada al cultivo, producción, comercialización y tráfico de drogas ha golpeado a todo el país y fracturado el tejido social y la convivencia comunitaria de los mexicanos en muchas regiones, dañando profundamente la confianza de la población hacia las instituciones. Este entorno dificulta, sin duda alguna, los programas y acciones de política pública orientadas a la prevención y atención integral de las adicciones.

Recuperar el tejido social y la certidumbre de un mejor futuro son los grandes retos del gobierno en lo inmediato.

A 50 años, la complejidad del fenómeno del consumo y la oferta de drogas es mucho mayor. Durante este tiempo, la lógica de los mercados cerrados ha cambiado dramáticamente. La globalidad económica, la carretera de la información sustentada en el mundo virtual, el internet y la informática han dado un nuevo rostro al mundo.

La concentración de la riqueza se ha agudizado, los referentes nacionales se han ido diluyendo, la reconstrucción de los imaginarios de cada grupo cambian 
vertiginosamente. En este sentido, la única certeza es el cambio y la indefinición de paradigmas sociales, políticos y culturales.

No obstante, la coyuntura de hoy en México y el mundo deviene en espacio histórico de oportunidad, para cambiar lo ineficaz, mejorar nuestra lectura de la realidad y construir nuevos paradigmas de interpretación social que nos permitan acercarnos con certidumbre a los grupos sociales y culturales cuyas necesidades y demandas tienen un espectro diverso y complejo.

Si no entendemos a la población, en sus múltiples formas de interacción y delimitación, difícilmente podremos incidir en sus circunstancias.

Tenemos que privilegiar y poner en el centro a las personas $y$, fundamentalmente, a las rezagadas y ausentes de los beneficios de la globalidad económica.

Bajo este marco, el consumo de drogas, como fenómeno social, cultural, familiar y psicológico individual, está inserto en la dinámica estructural de los últimos años. En el contexto de que las drogas son mercancías globales que procuran grandes ganancias y poder. La producción y comercialización de sustancias ilegales aprovecha los canales que la globalidad ha diseñado para la estructuración de los mercados mundiales.

Los consumos de drogas ilegales están orgánicamente relacionados hoy con lógicas de mercado, con la diversidad y disponibilidad de las sustancias, la baja percepción de daño de los consumidores y, también, con la construcción cultural que asocia la droga con la diversión, constituyéndose en una puerta de entrada.

No obstante, en este problemático entorno, el compromiso de Centros de Integración Juvenil, desde su origen, ha sido proteger la salud de los mexicanos, especialmente la de los niños, niñas y jóvenes.

Con renovada convicción, fortalecida a su vez por la celebración de nuestro 50 aniversario, seguiremos trabajando solidaria y responsablemente con el apoyo de la comunidad organizada, en favor de México. Reconocemos que, en esta labor, la investigación y el conocimiento del problema constituyen, hoy más que nunca, un ingrediente necesario de nuestros programas.

Contamos con la madurez que da la experiencia. Formamos recursos profesionales de primer nivel para atender integralmente el fenómeno de las adicciones. Contribuimos en la generación y divulgación del conocimiento científico. Trabajamos para y con la comunidad en beneficio de la población. Asimismo, procuramos la participación y el apoyo de la sociedad, a fin de que los programas y proyectos que implementamos cuenten con el soporte de la comunidad.

Como siempre, nuestro compromiso es con México. 